\title{
526 REMOVAL OF SOLUBLE TUMOR NECROSIS FACTORS RECEPTORS $1 / 2$ IN PATIENTS WITH METASTATIC SOLID TUMORS USING IMMUNE APHERESIS
}

${ }^{1}$ Ayala Tamir, ${ }^{2}$ Hagit Harati, ${ }^{2}$ Nethanel Asher, ${ }^{2}$ Ronen Stoff, ${ }^{2}$ Shirly Grynberg, ${ }^{3}$ Robert Segal, ${ }^{3}$ Adam Ostrowski, ${ }^{3}$ Lawrence Florin, ${ }^{2}$ Ronnie Shapira-Frommer*, ${ }^{4}$ Gal Markel. ${ }^{1}$ Sheba Medical Center, Israel, Ramat Gan, Israel; '2Sheba Medical Center, Ramat Gan, Israel; ${ }^{3}$ Immunicom Inc., San Diego, CA, USA; ${ }^{4}$ Rabin Medical Center, Petach Tikva, Israel

Background TNF $\alpha$ is a cytokine produced by immune cells and by tumor cells. The soluble forms of membrane TNF receptors $1 / 2$ (sTNF-R1/2) act as decoy to neutralize TNF $\alpha$, and are highly abundant in cancer patients. Elimination of sTNF-R1/2 may therefore unmask endogenous TNF $\alpha$, to presumably exert anti-neoplastic effects and reverse resistance to immune checkpoint inhibitors. Immune Apheresis (IA) is a procedure designed to specifically capture sTNF-R1/2 from plasma by passing it over an affinity column. Here we employed Immunicom's LW-02 Immunopheresis ${ }^{\circledR}$ device for removal of sTNF-R1/2 from plasma of cancer patients.

Methods In cohort A, patients with melanoma, RCC, NSCLC or TNBC refractory to standard therapy were treated with IA only. IA treatment of 2 plasma volumes was done $\mathrm{x} 3 /$ week, for three treatment cycles (4 weeks each) up to a total of 36 treatments. Cohort B patients currently receive concurrent IA and Nivolumab therapy $(240 \mathrm{mg}$ q2 weeks starting on week 5). sTNF-Rs removal and circulating inflammatory biomarkers were measured by immuno-assays, such as multiplex cytokine detection and mass cytometry. Pre- and post-treatment tumor biopsies were analyzed for tumor markers and TILs by immunohistochemistry.

Results Cohort A included six patients (3 Melanoma and 3 TNBC): three patients completed full study regimen, and three others were withdrawn due to clinical progression. AEs included chills (4/6), fever (2/6), anemia (6/6), central line thrombosis (1/6) and pulmonary embolism (1/6) All were Grade 2 except G3 anemia (1/6). There were no treatment related SAE's. sTNF-Rs levels were significantly reduced, followed by enhanced detection of TNF $\alpha$, and IFN $\gamma$ in some cases. In two patients, CD8 counts and PD-1 and PD-L1 expression were increased. Congruently, blood mass cytometry showed reduction in Treg subsets and differential increase of CD8 subsets following treatment.

Conclusions The use of Immunicom's LW-02 Immunopheresis ${ }^{\circledR}$ device in combination with Terumo BCT Spectra Optia Apheresis System is safe and efficient in the removal of sTNF-Rs from blood plasma. Subsequent immuno-assay analyses indicated formation of inflammatory response which may facilitate effects of immunotherapy, yet to be investigated in cohort B.

Trial Registration NCT04142931

Ethics Approval Sheba Medical Center Ethics Committee, 6136-19

Consent Written informed consent was obtained from the patient for publication of this abstract and any accompanying images. A copy of the written consent is available for review by the Editor of this journal

http://dx.doi.org/10.1136/jitc-2021-SITC2021.526 